\title{
Enhanced Trabeculectomy - The Moorfields Safer Surgery System
}

\author{
Peng Tee Khaw ${ }^{\mathrm{a}, \mathrm{b}} \cdot$ Mark Chiang, ${ }^{\mathrm{a}, \mathrm{f}} \cdot$ Peter Shah ${ }^{\mathrm{a}, \mathrm{c}, \mathrm{d}, \mathrm{g}} \cdot$ Freda Sii ${ }^{\mathrm{c}, \mathrm{g}}$. \\ Alastair Lockwood ${ }^{\text {a,e }} \cdot$ Ashkan \\ Khalilia,
}

aThe National Institute for Health Research Biomedical Research Centre at Moorfields Eye Hospital NHS

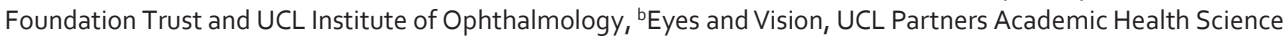
Centre, London, 'University Hospitals Birmingham NHS Foundation Trust, Birmingham, ${ }^{d}$ Centre of Health and Social Care Improvement, School of Health and Wellbeing, University of Wolverhampton, Wolverhampton, eUCL School of Pharmacy, London, UK; fDepartment of Ophthalmology, Royal Brisbane and Women's Hospital, Brisbane, Qld., Australia, ${ }^{9}$ Birmingham Institute for Glaucoma Research, Institute of Translational Medicine, Birmingham, UK.

\begin{abstract}
Trabeculectomy with anti-fibrotic treatment is still the most popular incisional procedure for glaucoma filtration surgery (GFS) worldwide. The advent of anti-fibrotic agents reduced failure due to scarring but resulted in increased complications. Advances in trabeculectomy surgery have been driven by the need to minimise the risk of: (1) complications and (2) surgical failure. This chapter cov- ers preoperative, intraoperative and postoperative strategies, which improve the outcome of GFS. Strategies to reduce the risk of complications centre on the prevention of postoperative hypotony by minimising the risk of overdrainage, postoperative wound leaks and poor bleb morphology. Surgical techniques to reduce the risk of postoperative fibrosis by the use of anti-fibrotic agents (including mitomycin C) are discussed in detail. These techniques are based on a combination of considerable clinical experience, observation and laboratory research. The need to address pre-, intra- and postoperative issues in each individual patient is emphasised. These changes are embod- ied in the system we call the 'Moorfields Safer Surgery System'. The use of these strategies has consid- erably reduced the incidence of major complications including hypotony, cystic blebs and endophthalmitis in practices around the world. Most of these techniques are simple, require mini- mal equipment and can be easily mastered. They are associated with an improvement in overall outcome and it is hoped that this chapter will help the reader benefit from these advances.
\end{abstract}

Copyright $@ 2012$ S. Karger AG, Basel

Trabeculectomy, first introduced in the 1960s, has become the gold standard for many types of glaucoma and is still the most commonly performed glaucoma filtration surgery (GFS). The operation of trabeculectomy aims to create a permanent drainage outflow channel for aqueous humour, connecting the anterior chamber (AC) to the sub-Tenon's space. Failure of trabeculectomy can occur 
as a result of scarring at the site of the new drainage channel with subconjunctival fibrosis and scarring at either the level of the scleral flap or the ostium. However, traditional trabeculectomy surgery has previously been associated with a signifi- cant risk of complications including vision-threatening hypotony, bleb-related problems and endophthalmitis. Recently, based on clinical observation and experimental research, simple but significant modifications to the original trabe- culectomy techniques have evolved, which have considerably improved the success rates and reduced the complication rates of trabeculectomy, some of which have been embodied in a system we evolved known as the Moorfields Safer Surgery System.

The advent of trabeculectomy revolutionised GFS compared to previous fullthickness procedures [1]. The original description of trabeculectomy involved the use of a superior rectus suture, a limbal-based conjunctival flap (LBCF) and a deeper scleral flap with pre-placed sutures. The aim of the operation at that time was to excise a block of Schlemm's canal and trabeculum, allowing free aqueous drainage into the exposed ends of the canal of Schlemm, hence the name trabe- culectomy. Originally, it was never intended to make a fistula to result in a drainage bleb, which is interesting bearing in mind that in the initial concept there was no drainage bleb with contemporary nonpenetrating surgical techniques. However, the presence of a drainage bleb tended to be associated with an increased success rate. Trabeculectomy then began to be regarded as filtration surgery, and more attention was focused on surgical techniques, which facilitated the creation of diffuse drain- age blebs.

The advances in trabeculectomy were driven by the need to minimise the risk of: (1) complications, including postoperative hypotony, infection and bleeding, by preventing overdrainage, postoperative wound leaks and poor bleb morphol- ogy, and (2) surgical failure, by reducing postoperative fibrosis (using anti-fibrotic agents).

The variation in the current practice (preoperative strategies, intraoperative tech- niques and postoperative management) of GFS, diverse case-mix and different suc- cess criteria account for the variable results published in the literature, with 'success' rates ranging from 71 to 100\% [2-11]. In the Tube Versus Trabeculectomy (TVT) study, the cumulative probability of failure in the trabeculectomy group was $30.7 \%$ at

3 years and $46.9 \%$ at 5 years [3]. However, high success rates and low complication rates can be achieved when meticulous attention to preoperative risk stratification, intraoperative techniques and postoperative management is employed [ref]. Our success rate with mitomycin C (MMC)-augmented trabeculectomy is $96.7 \%$ [intraocular pressures (IOP) $\leq 21 \mathrm{mmHg}$ ] at 3 years in a complex case mix. [10]. Other surgeons using our system on lower risk patients have had good success rates with $100 \%$ IOP $<21 \mathrm{mmHg}$ and low complication rates such as flat AC (1.8\%) and hypotony (1.5\%) [11]. The system has also achieved good success and safety in normal tension glaucoma patients and paediatric glaucoma patients [ref]. 
This chapter covers the intraoperative techniques of GFS and specific strate- gies to minimise the risk of intra- and postoperative complications. Postoperative management, as well as the management of postoperative complications, is covered in other chapters.

\section{Surgical Technique}

\section{Filtration Area \\ Positioning}

GFS should be performed superiorly, fully protected by the upper lids. A peripheral iridectomy placed at 12 o'clock is also covered by the lid, and does not give rise to diplopia. Interpalpebral or lower fornix drainage blebs have up to a 10 times greater incidence of inflammation and endophthalmitis, particularly if anti-fibrotic agents are used (fig. 1). Recurrent subconjunctival haemorrhage has been reported more commonly with inferior drainage blebs, together with bleb discomfort and pain. It is important to position the bleb with maximal upper lid coverage. Other surgical pro- cedures such as tube drainage surgery should be considered if this is not possible.

\section{Corneal Traction}

\section{Suture}

Superior rectus traction sutures may cause globe perforation, retrobulbar haemor- rhage, postoperative ptosis, superior rectus haematoma and subconjunctival haem- orrhage. Subconjunctival haemorrhage or superior rectus haematoma may trigger an excessive wound healing response in trabeculectomy. The use of superior rectus trac- tion sutures was associated with reduced trabeculectomy success in the CAT152 anti- body trial [12]. The corneal traction suture technique $[13,14]$ reduces the potential conjunctival damage and bleeding caused by a superior rectus traction suture. The vec- tor force of the corneal suture is superior to that achieved with a superior rectus suture. The disadvantages of the corneal traction suture include the small risk of placing the suture too deeply and penetrating the $\mathrm{AC}$ (particularly in eyes with thin corneas) and the chance of placing the suture too superficially with subsequent cheese-wiring and loss of traction. A variety of sutures can be used, and we generally use a spatulated 7-0 black silk suture on a $3 / 8$ curved needle (fig. 2). This can be anchored on 'pulley' set up using either a special speculum or curved artery clip fixed to the drape.

\section{Types of Conjunctival}

Incision 
There are 2 principal types of conjunctival incision in GFS: (1) Limbal-based conjunctival flaps (LBCF) with the incision deep in the conjunctival fornix and the base at the limbus, and (2) fornix-based conjunctival flaps (FBCF) with the incision at the limbus and the base at the fornix. There may be increased postoperative wound leaks using FBCF as initially noted by surgeons converting from LBCF to FBCF. However this is no longer the case with modern closure techniques. The use of FBCF may offer advantages particularly important in glaucomas associated with poor surgical success [15]. The pros and cons of both FBCF and LBCF are summarised in table 1. 
Fig. 1. Endophthalmitis from an interpalpebral bleb.

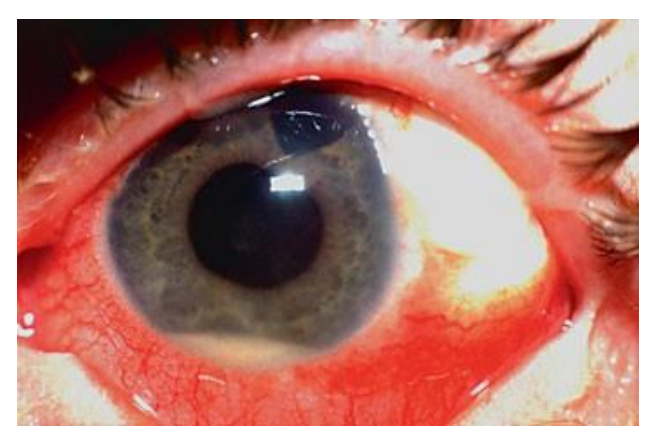

Fig. 2. Corneal traction suture provides better exposure and no secondary subconjuctival haemorrhage.

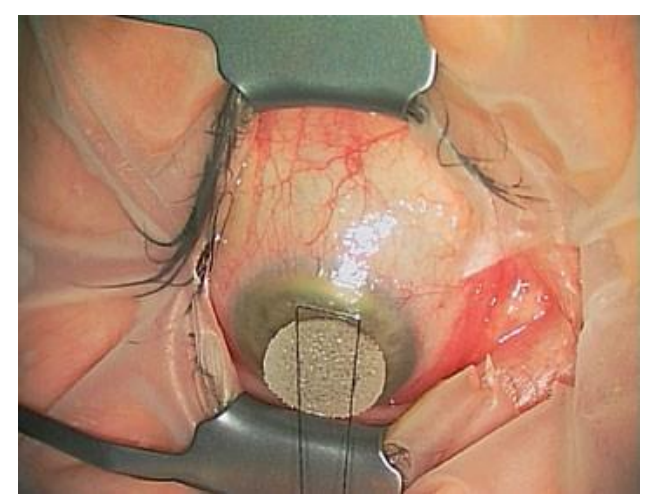

Fig. 3. Khaw cystic bleb hypothesis. Cystic bleb dissection revealing predisposing factors for cystic bleb formation - a ring of scar tissue and anteriorly directed aqueous flow.

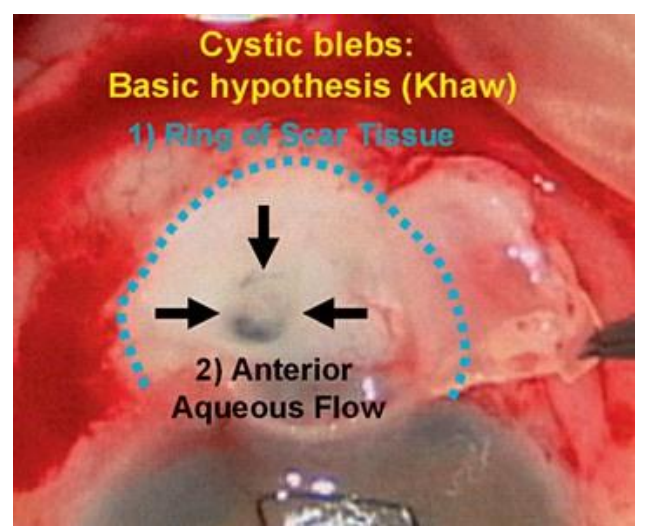

The widespread use of anti-fibrotic agents has increased the differences between the two approaches. Based on clinical observation, we proposed two phenomena necessary for cystic blebs: (1) anterior aqueous drainage and (2) a peri-bleb ring of scar tissue coined the 'ring of steel'. LBCF was more likely to result in a cystic bleb when the con- junctival incision was not posterior enough. It was technically easier to achieve a larger 
Table 1. Fornix-based and LBCF: pros and cons

\begin{tabular}{|c|c|c|}
\hline Type of conjunctival flap & Fornix based & Limbal based \\
\hline Technical difficulty & Easier and faster to perform & $\begin{array}{l}\text { More difficult and takes longer to } \\
\text { perform }\end{array}$ \\
\hline Exposure of operative field & $\begin{array}{l}\text { Enhanced exposure allowing } \\
\text { good visualisation of } \\
\text { sclerostomy and easier } \\
\text { 'releasable' suture placement }\end{array}$ & $\begin{array}{l}\text { Reduced exposure, less visualisation } \\
\text { of sclerostomy and reflected } \\
\text { conjunctiva can make placement of } \\
\text { releasable sutures difficult }\end{array}$ \\
\hline Area to be dissected & Smaller & Larger \\
\hline Anti-fibrotic application & $\begin{array}{l}\text { Need great care } \\
\text { May need more sponges }\end{array}$ & Easier \\
\hline Re-operation & Easier & $\begin{array}{l}\text { More difficult due to extensive } \\
\text { scarring }\end{array}$ \\
\hline Bleb morphology & $\begin{array}{l}\text { More diffuse posteriorly } \\
\text { draining blebs }\end{array}$ & $\begin{array}{l}\text { May get more cystic blebs and ring } \\
\text { of steel with more anterior drainage } \\
\text { if incision not very posterior }\end{array}$ \\
\hline $\begin{array}{l}\text { Conjunctival wound } \\
\text { leakage }\end{array}$ & $\begin{array}{l}\text { May have higher incidence if } \\
\text { water-tight conjunctival } \\
\text { closure technique is not used } \\
\text { - published results variable }\end{array}$ & $\begin{array}{l}\text { May have lower incidence using } \\
\text { conventional closure }\end{array}$ \\
\hline
\end{tabular}

area of MMC treatment with a FBCF, which prevented the ring of steel (fig. 3). We studied the postoperative bleb morphology in young patients undergoing trabeculectomy with high dose MMC and reported significantly more cystic blebs using LBCFs (90\%) compared to FBCFs (29\%), with a marked reduction in bleb-related complications including leakage, blebitis and endophthalmitis [16]. FBCF has now been widely adopted due to the dramatic reduction in incidence of cystic blebs as experienced by individuals such as Paul Palmberg in Miami.

\section{The Conjunctival Flap}

Handling of the conjunctiva must be gentle and kept to a minimum to avoid buttonholing, particularly if anti-fibrotic agents are used. If the tissues are particularly thin and prone to tearing, stromal hydration can be performed at the start of FBCF by injecting balanced salt solution (BSS) via a blunt cannula underneath the conjunctiva and Tenon's into the region of the future bleb. This manoeuvre hydrates the tissues, making them thicker and easier to handle. In both LBCF and FBCF, haemostasis is important and achieved by using minimal wet-field diathermy (unipolar or bipolar) to the sclera to prevent tissue damage and shrinkage. Lidocaine $2 \%$ and adrenaline 1:200,000 (Astra Zeneca, UK) can also be used, and this has the advantage of 
enhancing hydrostasis and minimising the local pain receptor feedback loop result- ing in further local inflammatory cytokine release. Topical apraclonidine $1 \%$ is also very useful to achieve haemostasis before and after incising the conjunctiva [17].

\section{Fornix-Based Approach}

A conjunctival incision of between 6 and $8 \mathrm{~mm}$ is needed. A relieving incision is not required and increases tissue trauma and risk of wound leakage. A conjunctival/ sub-Tenon's pocket measuring approximately $10-15 \mathrm{~mm}$ posterior and wide is constructed by careful dissection using blunt scissors. Care is taken to accurately identify and dissect into the supra-scleral sub-Tenon's plane. The essential step in this process is to identify and dissect through and posterior to the Tenon's ligamentous insertion. When dissecting over the superior rectus tendon, the conjunctiva is lifted while cutting the attachments to avoid the tendon itself.

\section{Limbal-Based Approach}

If this is used, the incision should be made as posteriorly as possible into the fornix to avoid restriction of aqueous drainage due to scarring (ring of steel). Diffuse blebs can be achieved with LBCF with a very posterior incision. The conjunctiva and Tenon's should be entered in separate layers to minimise the chance of damaging the superior rectus muscle. An incision length of at least $10 \mathrm{~mm}$ is needed to provide adequate exposure.

\section{Scleral Flap}

The scleral flap provides resistance to aqueous outflow and prevents hypotony. It also acts as a safety valve to minimise IOP fluctuations or spikes. There are several types of scleral flap - the most common being rectangular or triangular in shape. Studies on the influence of scleral flap position and dimensions in experimental trabeculectomy have shown that good IOP control can be achieved with a variety of scleral flap types [18]. We favour the construction of a rectangular partial-thickness scleral flap ( $\sim 50 \%$ thickness) and outline a $3.5 \times 4.5 \mathrm{~mm}$ flap with a blade, followed by lamellar dissection anteriorly just into clear cornea using a scleral pocket knife. Alternatively, a scleral pocket can be made after an initial incision, before performing the two side incisions (fig. 4). The side incisions are cut approximately $85-90 \%$ to the limbus to reduce anterior aqueous drainage and encourage posterior aqueous flow, resulting in a more diffuse non-cystic bleb (fig. 5). If the sides of the scleral flap are dissected all the way to the limbus, there is an increased risk of hypotony [18].

\section{Preventing Scleral Flap Problems}

Thin scleral flaps must be avoided because they increase the risk of flap dehiscence, formation of full-thickness scleral blow-holes with aqueous jet formation and cheesewiring of the flap sutures; all of which lead to reduced flap resistance with unprotected aqueous outflow and subsequent hypotony. This is very important in eyes with low scleral rigidity (e.g. buphthalmos and myopia) and those with anti-fibrotic 
Fig. 4. Scleral pocket being dissected using pocket knife.

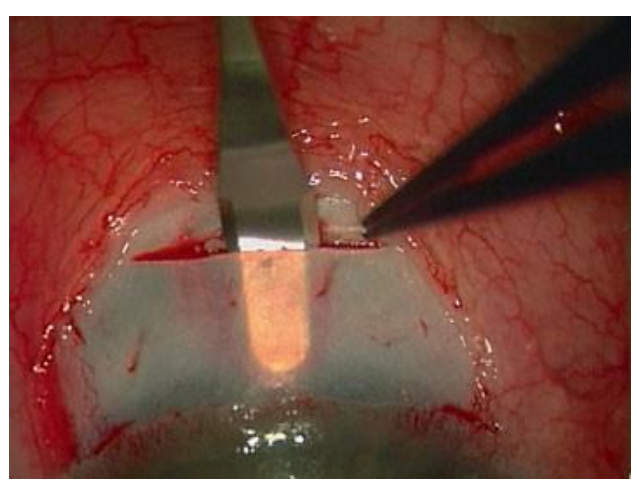

Fig. 5. Limited side cuts to scleral flap to encourage posterior flow and diffuse bleb.

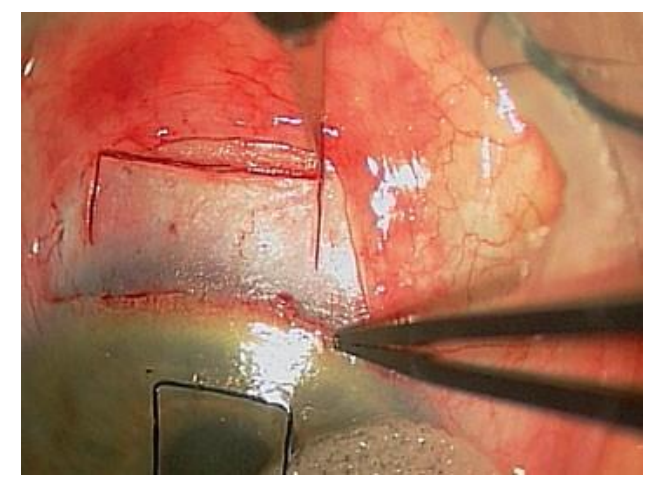

agent use because the conjunctival resistance may not rise for several weeks or even months after surgery. The position of the scleral flap should be chosen so that it does not involve large perforating aqueous veins, which may result in full-thickness blowholes in the scleral flap.

\section{Peri-Operative Use of Anti-Fibrosis Agents}

\section{Anti-Fibrotic Agent and Treatment Duration}

The choice of anti-fibrotic agent and treatment duration should be stratified according to patient risk factors for scarring (table 2) and complications (table 3). The Moorfields 'More Flow'anti-scarring regimen is an example of such a model (table 4). The regimen uses 3 min of MMC treatment and titrates the effect of MMC by varying the MMC concentration. Intraoperative 5-fluorouracil (5-FU; $50 \mathrm{mg} / \mathrm{ml}$ ) can be used in patients at lower risk of failure. Pharmacokinetic experiments have shown rapid uptake of anti-fibrotic agents applied on topical sponges over $3 \mathrm{~min}$, after which there is a plateau with relatively little effect seen for extra duration of application. In the 
Table 2. Risk factors for failure due to scarring after GFS

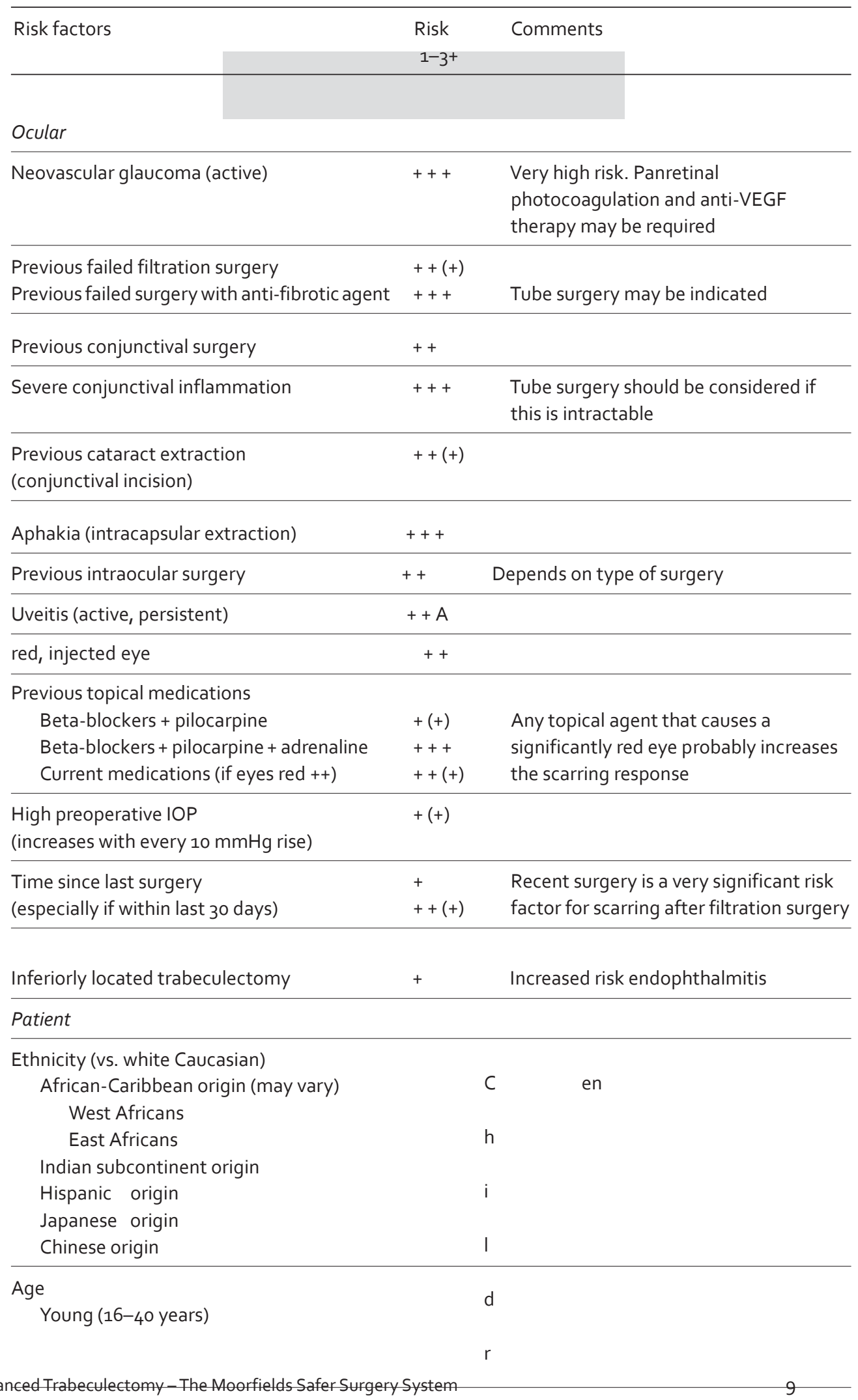




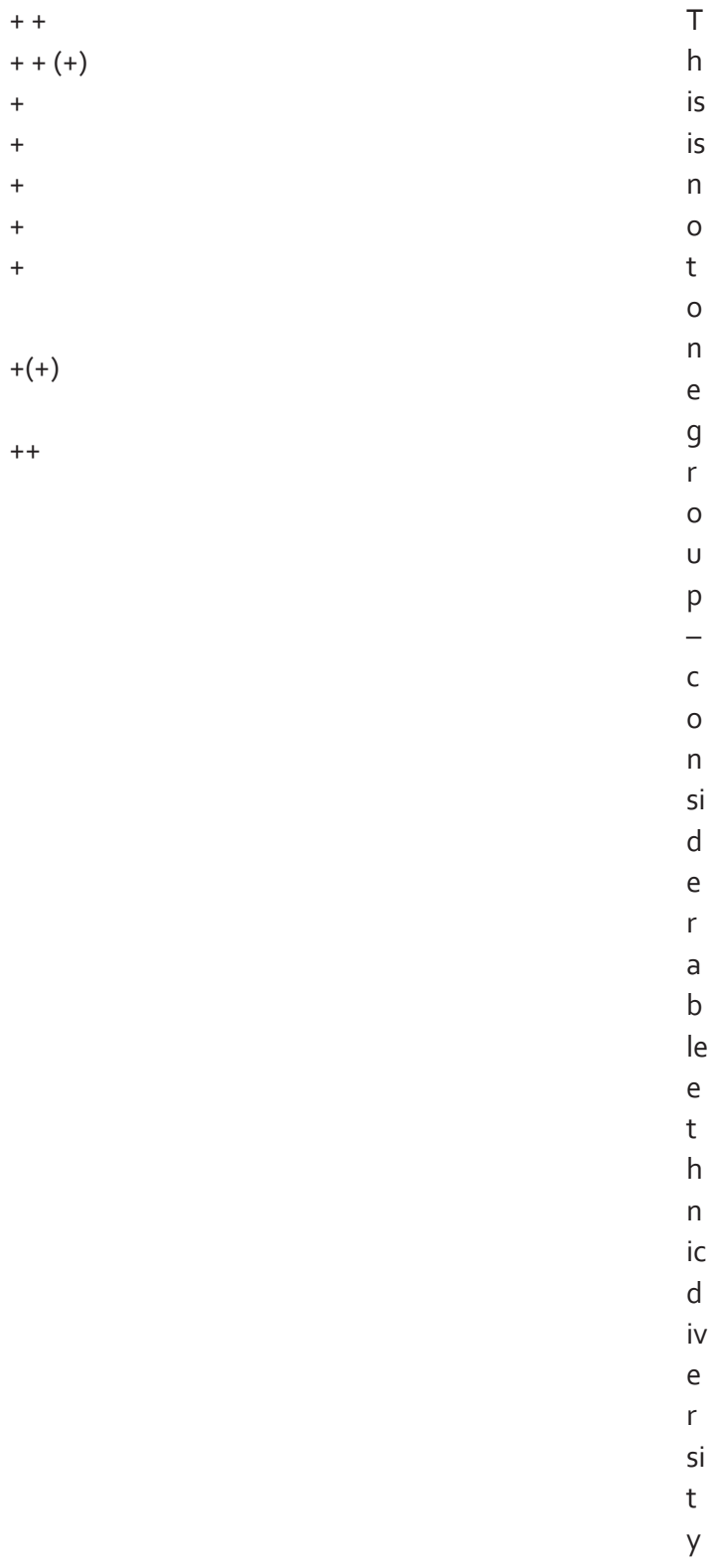

literature. Outcome more likely related to underlying condition.

p

c

o

n

si

d

a

b

le

e

t

h

n

ic

d

iv

e

r

si

$\mathrm{t}$

y

Unce

rtain,

differ

ent

repor

ts in 
Table 3. Possible risk factors for anti-fibrotic-related complications

Risk factors for complications

Thin or hyperelastic scleral tissue prone to collapse, e.g. myopia/buphthalmos/Ehlers-Danlos syndrome (significant risk)

Bleb placed in interpalpebral or inferior position (up to $10 \times$ risk)

Elderly patient

Primary surgery, no previous medications

Thin conjunctiva or sclera

Table 4. Moorfields Eye Hospital (More Flow) intraoperative single dose anti-fibrotic regimen v2011 (continuously evolving)

Lower risk patients (none or intraoperative $5-\mathrm{FU} 50 \mathrm{mg} / \mathrm{ml}^{1,2}$ )

No risk factors

Minimal topical medications (beta-blockers only)

Elderly (>70 years old)

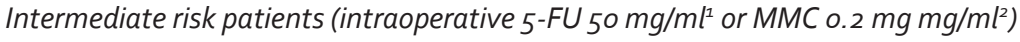

Chronic topical medications

Previous cataract surgery without conjunctival incision (capsule intact)

Several low risk factors

Previous conjunctival surgery e.g. squint surgery/detachment surgery/trabeculotomy

High-risk patients (intraoperative MMC $0.5 \mathrm{mg} / \mathrm{ml}^{2}$ )

Neovascular glaucoma 3

Chronic persistent uveitis

Previous failed trabeculectomy/tubes

Combined GFS/cataract extraction

Chronic marked conjunctival inflammation, e.g. pemphigoid, allergy ${ }^{3}$

Multiple risk factors

Aphakic glaucoma (a tube may be more appropriate in this case)

Lower target pressures would suggest a stronger agent was required.

${ }^{1}$ Intraoperative beta-radiation 1,000 cGy can also be used.

2 Postoperative 5 -FU injections can be given in addition to the intraoperative applications of antifibrotic agent.

${ }^{3}$ Anti-VEGF therapy such as bevacizumab can be considered in situations where neovascular disease or hypervascularity are marked components. 
application period from 1 to $3 \mathrm{~min}$, there can be considerable variation in the dose of anti-fibrotic agent delivered.

\section{Conjunctival/Sub-Tenon's Pocket}

In both LBCF and FBCF, a conjunctival/sub-Tenon's pocket measuring approximately10$15 \times 10-15 \mathrm{~mm}$ is constructed by meticulous dissection with blunt scissors to allow a large area for anti-fibrotic treatment. The dissection must be made into the supra-scleral sub-Tenon's plane. In cases of extensive scarring or when the Tenon's capsule has multiple layers (e.g. in African-Caribbean eyes), a Tookes knife can be used to dissect off Tenon's and expose the bare sclera.

\section{Sub-Tenon's/Sub-Scleral Flap Anti-Fibrotic Treatment}

Anti-fibrotic agent can be applied onto the bare sclera alone, or in combination with treatment under the scleral flap. The main concern against sub-scleral flap treatment was the fear of increased intraocular anti-fibrotic entry causing toxicity. However, there is reasonable pharmacokinetic and clinical data now to suggest that sub-scleral application is safe. If sub-scleral flap treatment is planned, anti-fibrotic agent is applied after constructing the scleral flap [19], but before any incision into the AC.

\section{Techniques of Application of Anti-Fibrotic Agents}

Variations in the technique used to deliver intraoperative anti-fibrotic agent may account for some of the variations in efficacy and complications seen in the literature. It is very important for individual users to maintain a consistent technique and to build up experience with one technique.

Conjunctival Clamp

Special non-crushing conjunctival clamps (e.g. -and-Kent.com T-clamp No 2-686 or MkII UCLB Instruments) may be helpful. These hold back the conjunctiva and maintain the conjunctival pocket (fig. 6). Clamp use may minimise antifibrotic agent contact with the conjunctival edge and facilitate optimal conjunctival healing to prevent postoperative wound leaks and bleb-related problems.

Anti-Fibrotic Agent Delivery

Many methods exist for delivering anti-fibrotic agent into the sub-Tenon's pocket. Medical grade polyvinyl alcohol (PVA) sponges are preferred to methylcellulose sponges because they maintain integrity and do not fragment [20]. In contrast, methylcellulose sponges fragment relatively easily, increasing the chance of leaving residual microdebris, causing foreign body granulomas [21].

The PVA sponges used as LASIK corneal shields can be cut in half or be folded before insertion into the pocket without edge contact (fig. 7, 8). Some surgeons prefer to use multiple small PVA sponges $\left(8 \times 2 \mathrm{~mm}^{3}\right)$, counted in and out of sub-Tenon's pocket. 
Fig. 6. Conjunctival T clamp for holding tissue away from anti-fibrotic agent.

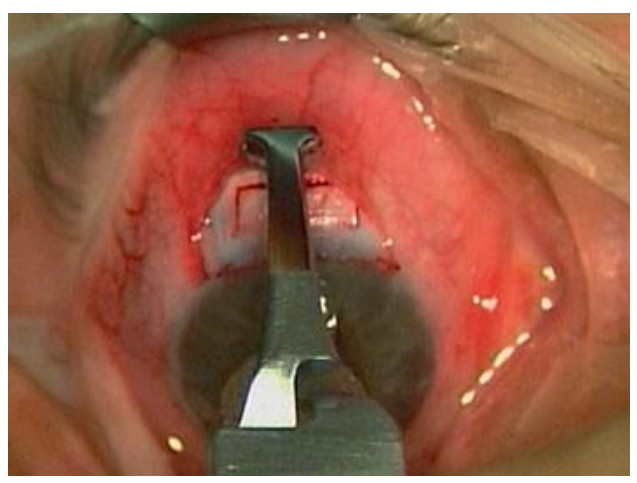

Fig. 7. Polyvinylalcohol sponges being folded.

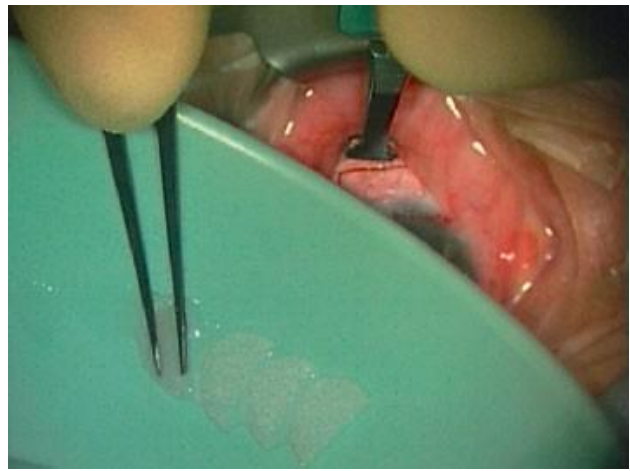

Fig. 8. Polyvinylalcohol sponge being inserted avoiding the cut edge of conjunctiva.

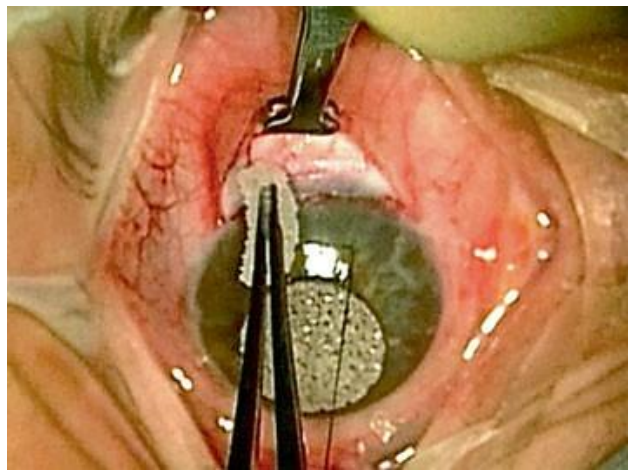

Anti-fibrotic treatment should cover the largest possible area to create a more dif- fuse non-cystic bleb and prevent the development of a posterior limiting scar (ring of steel). Immediately after sponge removal (count the sponges in and out), irrigation with $20 \mathrm{ml}$ of BSS is performed. 


\section{Function and Types of Scleral Flap Sutures}

The function of the scleral flap sutures is to secure the scleral flap and provide adequate tension so that the flap acts as an aqueous flow restrictor. The tension provided by the flap and sutures is particularly important when anti-fibrotic agents are used, as the scleral flap is the primary regulator of IOP until resistance builds up at the level of the conjunctiva/Tenon's, which may be many months after GFS. This tension is also important in eyes prone to postoperative AC shallowing, malignant glaucoma and/or hypotony. In these cases, the sutures should be tied tight to provide sufficient resistance to aqueous outflow. Several types of suture can be used, including interrupted sutures that can be lasered (laser suture lysis, LSL), releasable sutures that can be removed or adjustable sutures.

\section{Placement of Sutures}

In a rectangular scleral flap, the initial two sutures are placed at the posterior corners of the scleral flap, using a 10-0 nylon suture. Some sutures (e.g. 10-0; Alcon, Fort Worth, Tex., USA) are subjectively better for use as releasable or adjustable sutures. Having placed the initial two sutures, the need for further flap sutures can be assessed by inflating the eye through the paracentesis and observing the amount of aqueous outflow through the scleral flap. Pre-placing the scleral flap sutures is technically easier on a firm globe before intraocular entry, and the sutures can be tied rapidly during closure to shorten the duration of intraoperative hypotony.

\section{Principles of Scleral Flap Suture Removal}

The aim of placing and removing the scleral flap sutures is to control postoperative IOP by titrating the flow of aqueous through the scleral flap valve. In general, suture removal/lysis is avoided in the early postoperative period to prevent inadequate flap resistance and aqueous overdrainage leading to hypotony. This is a particular problem with adjuvant anti-fibrotic therapy. However, early suture removal (leading to an early high aqueous flow) may be required in some circumstances such as in young patients, African-Caribbean patients and patients with severe secondary glaucoma where early sub-Tenon's fibrosis with irreversible suboptimal bleb architecture can occur rapidly.

\section{Fixed Sutures and Laser Suture Lysis}

LSL was introduced in 1983 by Lieberman using an argon laser to lyse excessively tightened scleral flap sutures after trabeculectomy [22]. Since then, there has been much interest in LSL including the use of different lasers and power settings [2327] and the introduction of different types of suture lysis device/lenses. The most substantial IOP drop after LSL occurred during the first 2 weeks and was usually ineffective after the 4th postoperative week. LSL can be associated with significant 
complications including hypotony with shallow AC, progressive significant cataract, conjunctival perforation and conjunctival wound leak [28].

The delayed healing caused by MMC in trabeculectomy extends the clinically effective window period for LSL. Many studies demonstrated that LSL was effective beyond 2 months in some cases $[29,30]$. However, post-LSL hypotony remained sig- nificant and led to decreased vision. The safety of LSL was investigated in a large study by Macken et al. [31] which found significant flat AC with iridocorneal touch (13.1\%) and conjunctival leaks (9\%). Kapetansky [32] minimised post-LSL hypotony by delaying LSL after trabeculectomy with MMC by using digital massage to gauge the degree of healing. LSL after the 2nd postoperative month was suggested to pre- vent LSL-induced hypotony without adversely affecting the final IOP. In contrast, in eyes that had LSL within 10 days after trabeculectomy with MMC, the longterm IOP control was no different to the non-LSL group. One of the major concerns with LSL has been the effect of laser on the conjunctiva overlying the scleral sutures. Cases of conjunctival perforation resulting in persistent leak have been described $[28,33]$.

\section{Releasable Sutures}

Several years after the introduction of LSL, interest in releasable scleral flap sutures increased because of the technical and logistical difficulties of performing LSL [34]. In LSL, the scleral flap sutures can be obscured by subconjunctival haemorrhages, thickened Tenon's capsule or fibrosis [35]. The idea of releasable scleral flap sutures originated from Shaffer et al. in 1971 where externalised mattress sutures were used to prevent shallow AC following thermal sclerostomies [36].

The method described by Cohen and Osher [37] with a trimmed suture end on peripheral cornea, remained the most popular. Cases of endophthalmitis have been reported following trabeculectomy with MMC using this technique possibly due to the exposed suture end acting as a nidus for infection $[38,39]$.

Later modifications aimed to bury the externalised suture leaving no exposed free ends [40-42]. We reported the technique described in this chapter in 1996 [43]. It eliminates the free suture end, leaving all sutures buried but still accessible by the use of corneal grooves.

The use of releasable sutures has been found to significantly reduce postoperative hypotony and shallow AC compared to fixed scleral flap sutures. Reported complications include superior corneal epithelial abrasions and suture breakage on removal. There is no clear evidence that the use of releasable sutures improves GFS success $[34,41]$.

\section{Technique of Releasable Suture}

Two reverse-stepped clear corneal grooves are made to allow pre-placement of two releasable 10-0 nylon sutures in the scleral flap before blade entry into the AC. The suture is placed by passing the needle from just behind the limbus beside the scleral flap to emerge from the corneal groove. The needle is passed back into the corneal groove, through the partial-thickness scleral flap and emerges from the posterior 
third of the flap. The needle is then passed full-thickness through the corner of the scleral flap into the adjacent scleral bed at an angle of $45^{\circ}$, leaving a loop to be secured with a single triple-throw tie.

\section{Adjustable Sutures}

A subsequent adjustable suture technique allows transconjunctival adjustment of scleral flap tension postoperatively using specially designed forceps with smooth edges (fig. 9; Khaw adjustable forceps Duckworth-and-Kent.com, adjustable suture forceps No. 2-502 or MkII UCLB Instruments). The adjustable suture system (fig. 10) allows a gradual titration of IOP - and may allow more control than suture removal or massage $[44,45]$.

In summary, changes in area of anti-fibrotic treatment, conjunctival and scleral flap construction, and scleral flap sutures have led to a dramatic reduction of both shortand long-term complications (fig. 11). This has led to a reduction in cystic areas within the bleb from 90 to $29 \%$ as well as a reduction in the rates of blebitis and endophthalmitis [16]. Falls in complication rates have also been seen in the USA in lower risk populations from approximately 6 to $0.5 \%$ to date [Palmberg, pers. commun.].

\section{Fluid Flow Control}

\section{Paracentesis}

A paracentesis allows fine control of the AC in several intra- and postoperative situations: (1) To set the opening pressure of the scleral flap with precision after tying the flap sutures (IOP titration) - BSS is injected via the paracentesis while observing the emergence of fluid from the scleral flap. (2) Reformation (or decompression) of the AC, both intra- and postoperatively - BSS and/or viscoelastic can be used. (3) An infusion can be placed to maintain a continuous IOP, particularly in high-risk patients. (4) Control and washout of AC haemorrhage - viscoelastic can be injected to provide tamponade, and air and BSS for washout.

An oblique paracentesis placed in the inferior cornea parallel to the limbus is selfsealing. This technique minimises the risk of lens damage and is much safer if the $\mathrm{AC}$ is shallow.

\section{Infusion}

An anterior segment infusion (e.g. Lewicky, BD Visitec, N.J., USA) on a three-way tap can be used through the paracentesis to offer another level of IOP and AC control (fig. 12). The IOP and rigidity of the globe is maintained intraoperatively particularly in eyes with an increased risk of rapid choroidal effusion and haemorrhage (e.g. infants, high myopes, nanophthalmos, buphthalmos and Sturge-Weber syndrome). The IOP can be titrated using the infusion height to increase the accuracy of scleral flap closure and significantly reduce postoperative hypotony. 
Fig. 9. Adjustable suture forceps with special fine smooth tips for transconjunctival suture adjustment without tearing conjunctiva.

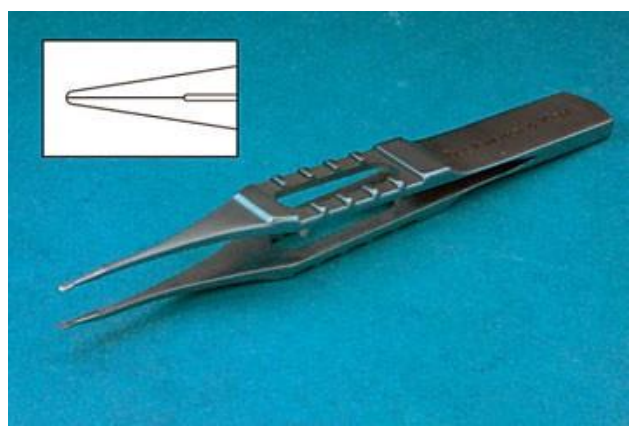

Fig. 10. Transconjunctival loosening of adjustable sutures without sudden fall in IOP.

Fig. 11. A diffuse bleb in the patient's right eye using a large area of treatment vs a smaller area of treatment with MMC.

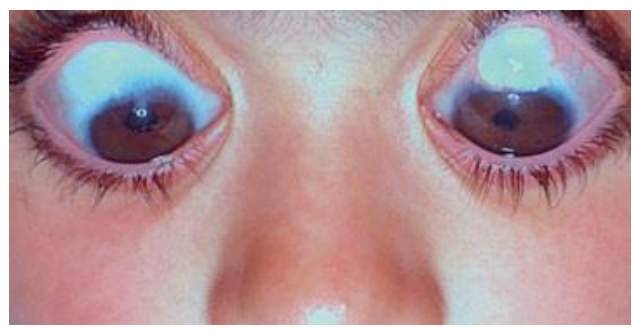

Fig. 12. $A C$ infusion to maintain IOP and gauge opening pressure of sclerostomy. Inserted with an oblique paracentesis in a phakic eye to minimise any risk of trauma to lens.

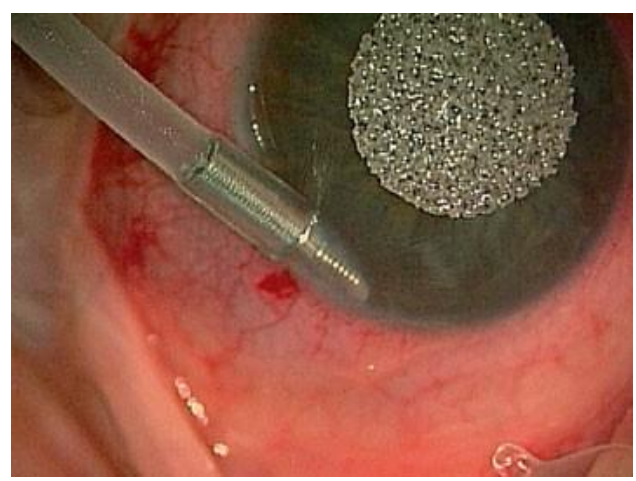




\section{Sclerostomy}

The sclerostomy can be created in various ways. It can be manually cut and removed with appropriate blade and scissors, or alternatively a special punch can be used.

\section{Placement of Initial Incision}

With the development of precision instrumentation, it is now possible to create an anterior corneo-scleral entry into the AC. An anterior incision reduces the risk of iris incarceration and bleeding from the iris root or ciliary body.

\section{Punch Sclerostomy}

A punch sclerostomy is the method of choice, and many punches are available. A sclerostomy measuring $0.5-2 \times 0.5-1.5 \mathrm{~mm}$ is adequate and provides optimum outflow control whilst minimising astigmatism and anterior aqueous flow [46]. Blade entry into the $\mathrm{AC}$ is performed anteriorly through clear cornea beneath the scleral flap. The punch is inserted to engage the full thickness of limbus. The punch should be aligned perpendicularly to ensure a clean non-shelved sclerostomy (fig. 13). We use a small $0.5-\mathrm{mm}$ diameter punch and repeat punching as required. A newer punch leaving a more oval hole and facilitating posterior flow and a diffuse bleb is now being developed (UCLB Instruments, London, UK).

\section{Manual Block Removal}

If a blade and scissors are used, it is difficult to cut a sclerostomy much smaller than $3 \times 1.5 \mathrm{~mm}$. The scleral flap is gently lifted with care not to cause a button-hole. The block is outlined to at least $90 \%$ depth before AC entry through the anterior edge. The rest of the block is dissected posteriorly to full-depth, using a blade or Vannas scissors to cut the base. Gentle traction is applied to the block during dissection to prevent damage to the iris and underlying structures. Problems with manual block removal include difficulty in cutting/dissecting anteriorly and the creation of a larger ostium causing shallow AC and hypotony.

\section{Peripheral Iridectomy}

A peripheral iridectomy (PI) is performed through the sclerostomy. The presence of a PI prevents iris incarceration and relieves pupillary block in some cases. The PI should be made relatively broad at the base and short in length to reduce the size of iris defect, minimising glare and monocular diplopia. Cutting the PI with scissors held parallel to the sclera facilitates this. The iris can be made to present through the sclerostomy without intraocular manipulation using an infusion, reducing iris trauma and the need for an assistant (fig. 14). 
Fig. 13. Small 0.5-mm titanium scleral punch to maximise flow control.

Fig. 14. Iris presenting through small sclerostomy with gentle pressure on back edge when infusion is used. No intraocular entry or manipulation is necessary.
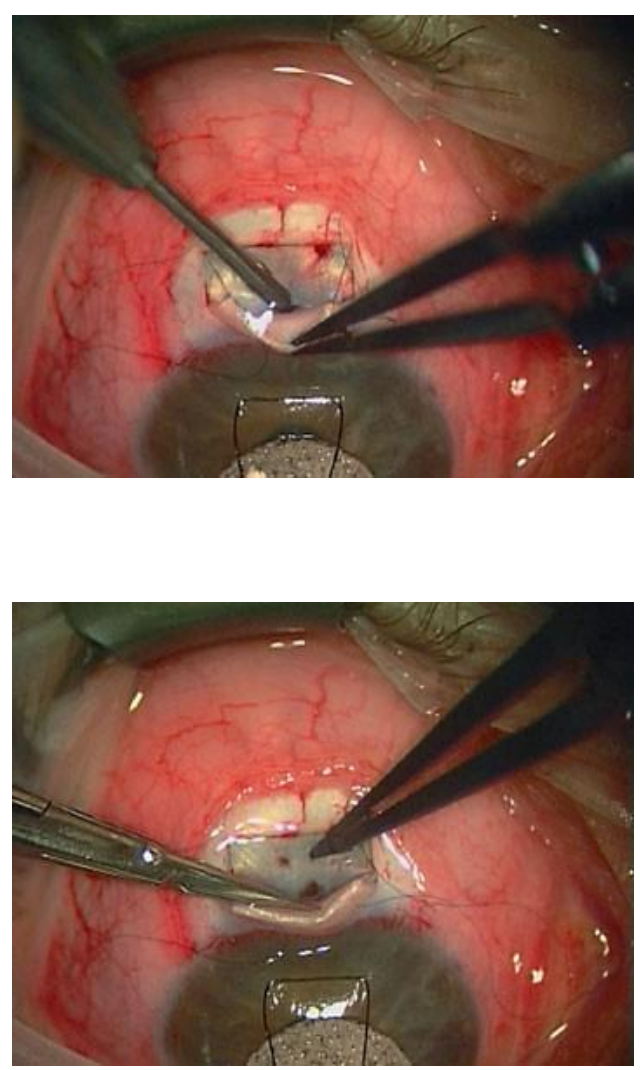

\section{IOP Titration}

After PI, pre-placed scleral flap sutures can be tied quickly to minimise the duration of hypotony. IOP titration is a key step in GFS where the opening pressure of the scleral flap is precisely set with the irrigation of BSS through the paracentesis or using an AC infusion. The point at which aqueous just emerges from the scleral flap is the opening pressure of the valve. Scleral flap sutures are tightened/loosened accordingly, and extra sutures placed as needed.

\section{Closure of Conjunctiva and}

Tenon's

Prevention of wound leaks is of paramount importance in FBCF surgery. Wound leaks lead to flattened bleb morphology and hypotony, increasing the risk of GFS failure. Therefore, great attention has been focused on reproducible water-tight clo- sure techniques. Traditional closure used single interrupted sutures at the ends of the conjunctival incision. Newer techniques include the use of edge pursestring sutures, 
interrupted horizontal mattress sutures or a suture with corneal grooves $[47,48]$. 'Corneal groove closure' involves creating a series of corneal grooves through which conjunctival sutures with buried knots can be placed (fig. 15). These techniques have virtually eliminated central conjunctival/Tenon's retraction, wound leaks and suture discomfort.

For LBCF, an absorbable suture (e.g. vicryl) is usually used for closure using interrupted or continuous suturing. Absorbable suture material is preferred for patient comfort and ease of management despite the theoretical increase in inflammation. A round-bodied rather than a spatulated needle is used (especially with MMC) to minimise the risk of conjunctival tears, cheese-wiring and suture tract leaks. Suture bites should include both the conjunctiva and Tenon's in single-layer closure to ensure a watertight wound. Following conjunctival closure, BSS is injected via the paracentesis to ensure patency of the scleral flap (observing the drainage bleb) and water-tight closure.

\section{Medications at End of Procedure}

Traditionally, a subconjunctival injection containing steroid and antibiotic is given $180^{\circ}$ away from the trabeculectomy site to prevent intraocular entry of these drugs. Mydriatics/cycloplegics such as topical atropine 1\% may be used. Advantages include relaxation of the ciliary muscle and pain relief, reduction of AC shallowing and malignant glaucoma (especially in short axial length eyes), stabilisation of the blood aqueous barrier (atropine mainly) and prevention of central posterior synechiae. Disadvantages include a dilated pupil, which may increase lens-corneal touch in shallow AC, and blurred vision.

\section{Prevention of Complications}

Scarring is the main cause of failure in trabeculectomy [49], and judicious use of the antifibrotic agents such as 5-FU and MMC prolongs functionality. However, these agents can also cause tissue thinning and necrosis. In one study, the annual incidence of bleb- related infection was reported to be as high as $1.3 \%$ [50]. In the TVT study, $5 \%$ of trabeculectomies had a bleb-related infection by 5 years and wound leaks were seen in $11 \%$, hypotony maculopathy in 3\% and suprachoroidal haemorrhage in 3\% [3]. Higher rates of hypotony have been reported, with hypotony seen in one third of trabeculectomies in one study [51] and delayed hypotony (after 6 months) seen in $42 \%$ in another [52].

However, complication rates can be minimised by appropriate strategies. This is reflected in our results with a very low rate of complications: endophthalmitis $(0.2 \%)$, blebitis $(0.2 \%)$, hypotony maculopathy $(0.2 \%)$, suprachoroidal haemorrhage $(0 \%)$ and wipe-out (0\%). 

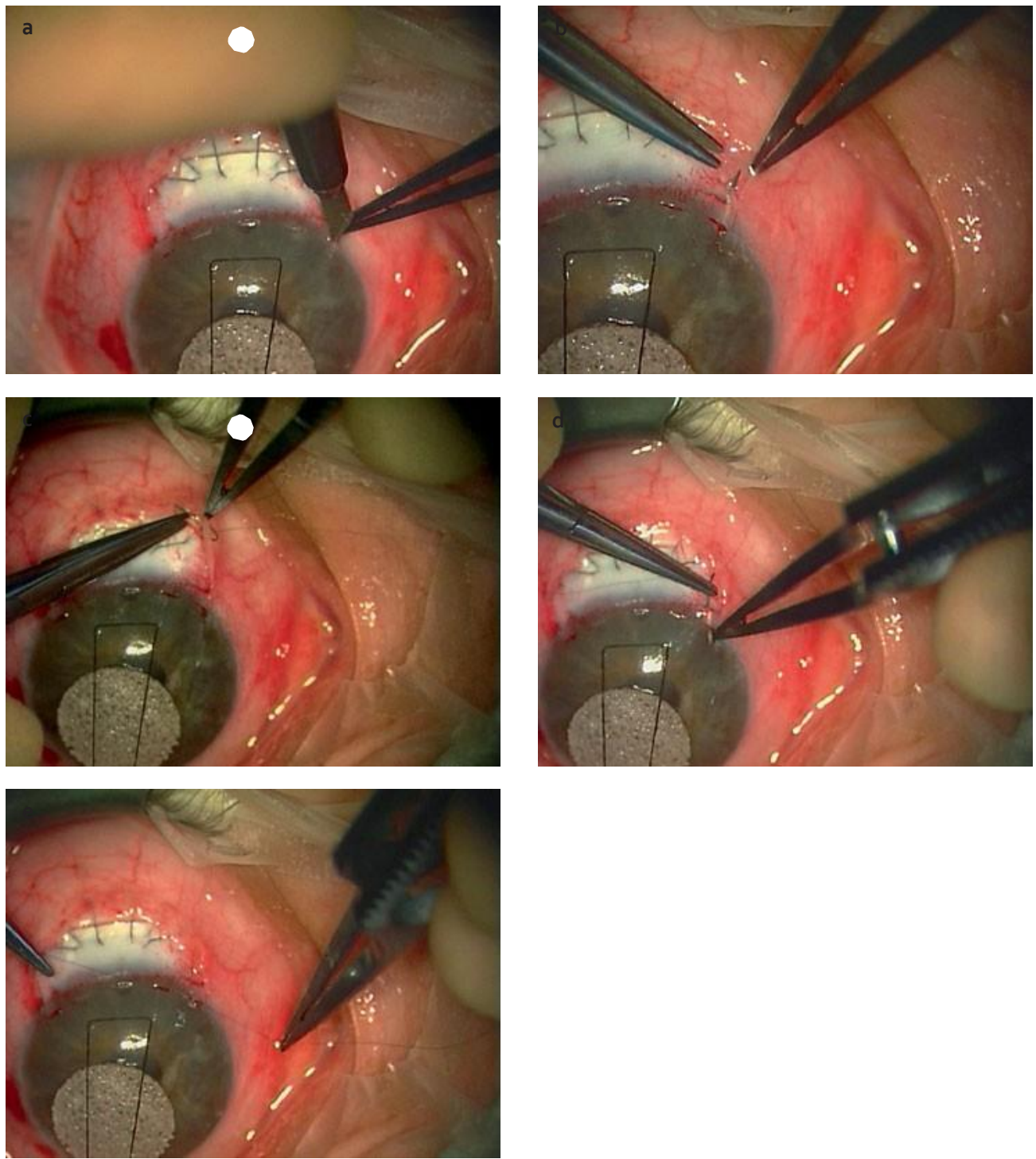

Fig. 15. Corneal groove creation (five grooves) for closure of fornix based conjunctival flap to minimise leakage and suture discomfort. a Two lateral purse strings sutures and 1-3 central mattress sutures. $\mathbf{b}$ Entry via corneal groove. c Purse string. $\mathbf{d}$ Exit via limbus and out via cornea. e Tie in corneal grove.

\section{Peri-Operative Complications}

\section{Conjunctival Tear}

Conjunctival tears can be a serious problem especially with the use of adjuvant antifibrotic agents. They most commonly occur during peritomy and sub-Tenon's dissection, especially in eyes with multiple previous surgery and adhesions. 


\section{Prevention}

The preoperative use of topical apraclonidine 1\% [17] or adrenaline significantly reduces tissue vascularity and bleeding, improving visualisation and control of the operative field. The handling of conjunctiva should be minimised. With thin tissues, BSS should be injected under the conjunctiva/Tenon's to thicken the tissues and hence allow easier handling (stromal hydration). This will also demarcate scarred tissue and open up tissue planes. During conjunctival/sub-Tenon's pocket construction, slow blunt dissection using very small cuts to divide adhesions and continuous reassessment of the plane minimises the risk of tearing the tissue.

Management

If a conjunctival tear occurs when adjuvant anti-fibrotic agent has not been used, it can be repaired by a simple purse-string vicryl suture on a vascular needle. If antifibrotic agents (especially MMC) are used, a patch graft using tissue without antifibrotic contact may be needed. This can be done by dissecting an attached flap of Tenon's capsule distant to the treatment area, and then rotating it underneath the conjunctiva to be sewn as a living patch underneath the tear.

\section{Conjunctival, Scleral and Iris Bleeding}

Bleeding must be minimised in GFS as blood is a potent stimulus for fibrosis. When bleeding occurs, prompt haemostatic measures should be employed.

\section{Prevention}

The prevention of intraoperative bleeding starts well before surgery with the discontinuation of aspirin, anticoagulants or herbal remedies (e.g. gingko biloba) if medically feasible. The use of topical apraclonidine $1 \%$ or adrenaline at time of surgery effectively reduces tissue vascularity. Handling of tissues (whether extraocular or intraocular) should be minimised. Control of blood pressure and use of general anaesthesia can reduce bleeding in high risk cases.

\section{Management}

Gentle wet-field diathermy (either bipolar or unipolar) can be used to control bleeding. The lowest possible power setting must be used to prevent tissue shrinkage. When rapid bleeding occurs from the PI, the pre-placed scleral flap sutures are tied and the IOP raised via the paracentesis to provide tamponade. Bleeding usually stops quickly. Then, the scleral flap can be loosened to allow gentle irrigation and AC washout. Larger haemorrhages may be controlled by raising the IOP with viscoelastic or AC infusion.

\section{Scleral Flap Damage}

Damage to the scleral flap including tearing or button-hole can occur during flap construction (before sclerostomy) or after sclerostomy. 
Prevention

Scleral flap damage is prevented by avoiding a thin flap which predisposes to cheesewiring and tearing, and reducing the amount of scleral flap manipulation.

Management

If the scleral flap is damaged during construction/dissection, further surgery should continue at a new site with undamaged sclera. After sclerostomy creation, minor flap damage may be repaired with a 10-0 nylon suture. In severe damage where the sclerostomy cannot be secured, an autologous or donor scleral patch, or processed pericardial graft may be sewn or glued with fibrin glue onto the operation site to regain globe integrity.

\section{Suprachoroidal Haemorrhage}

Suprachoroidal haemorrhage may occur in any eye where the AC is suddenly decompressed and shallowed (e.g. during sclerostomy creation), or where there is uncontrolled blood pressure or chronic obstructive pulmonary disease.

Prevention

The principle of preventing intraoperative SCH rests with the maintenance of IOP, whilst preventing sudden IOP reductions. The preoperative identification of highrisk eyes (e.g. young patient, large eyes, small eyes, Sturge-Weber syndrome) combined with modifications in surgical strategy are the critical steps in preventing this potentially blinding complication. Patients should stop medications that predispose to bleeding. Strategies to maintain intraoperative IOP include pre-placed scleral flap sutures, injection of viscoelastic into the AC before intraocular entry and the use of an AC infusion. The use of a sclerostomy punch as opposed to block excision also offers improved IOP control. Choroidal vasoconstriction induced by hyperventilation under general anaesthesia may also be a helpful measure. In nanophthalmic eyes, scleral decompression with the use of scleral windows may be required together with constriction of the choroidal bed with topical apraclonidine $1 \%$ before intraocular entry. Similarly, postoperative SCH is prevented by prevention of hypotony (e.g. use of viscoelastic or $\mathrm{C}_{3} \mathrm{~F}_{8}$ in aphakic eyes) and avoidance of Valsalva manoeuvres.

Management

If SCH is suspected, all wounds should be closed rapidly to ensure ocular integrity. The posterior segment is assessed to confirm the diagnosis. If SCH is peripheral and not affecting the macula, then a conservative approach is recommended. If SCH is extensive, drainage can be performed before the blood clots through one or more sclerostomies, providing that one has secured IOP control via an AC infusion.

Decompression Retinopathy

Retinal haemorrhages have been reported after rapid IOP reduction during GFS $[53,54]$. A sudden and substantial drop in IOP has been hypothesised to cause 
retinal vascular dysregulation and forward movement of the lamina cribrosa, leading to blockage of axonal transport and compression of the central retinal vein [55, 56].

Decompression retinopathy is prevented by gradual reduction of IOP using the paracentesis before intraocular entry and the use of an infusion. The use of general anaesthesia allows the IOP to be slowly reduced before the eye is entered.

\section{Vitreous Loss}

Vitreous loss during trabeculectomy occurs when the lens/zonule complex is inadvertently damaged during the PI, which is performed too posteriorly. The presence of vitreous in the AC will lead to potential blockage of the ostium and can increase the risk of GFS failure.

\section{Prevention}

Preventative measures are as follows: (1) create sclerostomy anteriorly; (2) avoid very basal PIs; (3) note irido-lenticular instability preoperatively and consider alternative procedure (e.g. tube surgery) if high risk of vitreous prolapse.

Management

Management consists of: (1) anterior vitrectomy, and (2) postoperative subconjunctival 5-FU.

\section{Postoperative Complications}

\section{'Wipe-Out' of Visual Field}

This is thought to occur more frequently in patients with advanced visual field loss, particularly if the field loss is within $10^{\circ}$ of fixation, although large-scale studies have not provided good supportive evidence.

\section{Prevention}

It may be useful to check the IOP a few hours after GFS to detect and treat any IOP spike. Intra- and postoperative hypotony, and systemic hypotension, all of which may compromise an already damaged optic nerve, should be avoided. GFS under general anaesthesia may avoid the IOP rise associated with some local anaesthetic techniques.

\section{Infection}

Blebitis and endophthalmitis are potentially blinding emergencies. Patients should seek ophthalmological attention immediately if they develop a purulent discharge. All postoperative patients should receive instructions regarding the risk of infection. 
Prevention of Early Infection

Measures to prevent early infection are: (1) identification of high risk patients (e.g. ocular surface disease and blepharitis) and management preoperatively; (2) use of

povidone-iodine to prepare the skin, lids, lashes and conjunctival sac; (3) minimise lashes in the operative field (use of steri-strips); (4) bury all sutures including releas-

able sutures; (5) patient education regarding the importance of hand washing and avoidance of contact with infected wounds.

Prevention of Late Infection

Late infections are prevented by avoiding blebs with poor/cystic morphology and man-

aging ocular surface disease. (1) Avoid excessive treatment with anti-fibrotic agents. (2).

Avoid thin scleral flaps, which predispose to blow-holes and necrosis (especially with MMC). (3) Use large areas of anti-fibrotic treatment to produce more diffuse non-cystic blebs. (4) Position bleb under the upper lidin a position with maximal cover. The blebitis/ scleritis and infection rate may be $5-10$ times higher with blebs unprotected by the upper

lid. Carry out tube drainage surgery or other therapies instead of inferiorly placed blebs.

\section{Wound Leak}

Conjunctival wound leaks must be avoided as they make further bleb manipulation difficult. Aqueous leakage decreases bleb height and leads to increased subconjunctival fibrosis and bleb failure.

Prevention

Preventative strategies include: (1) smaller conjunctival incision (in both FBCF and LBCF); (2) prevention of conjunctival tears and button-holes by minimising tissue handling and the use of round vascular (BV) needle; (3) in FBCFs, meticulous conjunctival wound closure; (4) in LBCFs, place conjunctival incision as posteriorly as possible; (5) careful conjunctival edge protection during anti-fibrotic application and the use of a non-crushing clamp; (6) identification of patients with poor wound healing ability (e.g. vitamin C deficiency and alcohol dependency).

\section{Bleb Overdrainage}

The use of anti-fibrotic agents (especially MMC) has increased the incidence of hypotony due to overdrainage. Most hypotony (IOP $<6 \mathrm{mmHg}$ ) in the early postoperative period settles without intervention. Hypotony becomes clinically significant when it persists with shallow AC, choroidal detachment or hypotony maculopathy - surgical intervention is then indicated.

Prevention

Hypotony due to aqueous overdrainage is commonly seen in eyes with low scleral 
rigidity (e.g. young patient, myopia, collagen abnormality). Preventative strategies include: (1) tight scleral flap closure with extra sutures as indicated by IOP titration; 
Fig. 16. Bleb oozing aqueous secondary to cystic change.

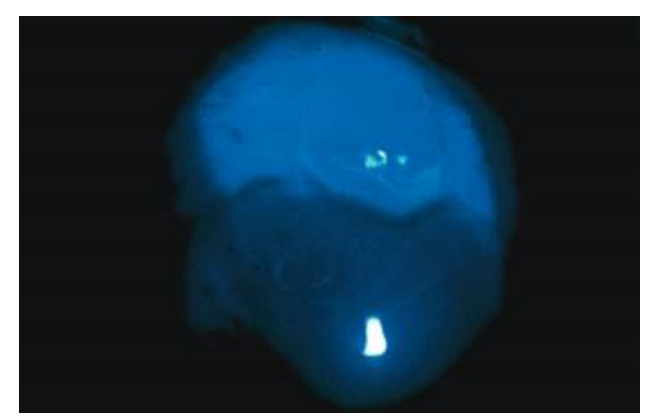

(2) avoid small or thin scleral flaps which may not provide adequate resistance to aqueous outflow; (3) do not release/lyse scleral flap sutures too early. With MMC, suture removal may result in hypotony even months after GFS. It is preferable to per- form bleb massage rather than removal of the releasable suture. (4) Avoid high dose MMC in patients prone to postoperative hypotony.

\section{Choroidal Effusion}

Choroidal effusions are common in hypotonous eyes following GFS. The collection of fluid (high protein content) in the suprachoroidal space is produced by transudation from leaking capillaries in the choriocapillaris. The measures to prevent choroidal effusion are the same as those for hypotony due to overdrainage.

\section{Ciliary Body Shutdown}

Patients with uveitis and patients with juvenile open angle glaucoma are at risk of developing ciliary body shutdown, which can be prevented by preoperative optimisation of intraocular inflammation. The management involves frequent topical and/or systemic steroids.

\section{Late Bleb Leak}

Bleb leaks can occur months or years after GFS, especially with adjuvant anti-fibrotic agents such as MMC. The leak may be focal or diffuse with 'sweating' areas (fig. 16). Avoiding thin scleral flaps reduces the risk of formation of blow-holes and anterior aqueous flow, which increase the risk of cystic bleb formation. Also, the use of anti-fibrotic agents must be titrated according to patient risk factors to avoid excessive treatment.

\section{Malignant Glaucoma/Aqueous Misdirection}

In malignant glaucoma/aqueous misdirection, the aqueous is directed posteriorly into the vitreous cavity with restriction of anterior flow by the anterior hyaloid face. Patients usually present with shallow AC and raised IOP several days after GFS. Anteriorly rotated ciliary processes have been observed to press against the lens equator and prevent anterior aqueous flow. The anterior hyaloid may be abnormally positioned, plugging spaces 
between ciliary processes. An acute episode of pupil block or SCH can sometimes give a similar picture.

Prevention

Eyes with reduced axial length (and/or increased posterior scleral thickness and uveal

effusion) must be identified preoperatively using A and B scan ultrasonography. Prophylactic sclerotomies may be required in nanophthalmos. Aqueous overdrainage must be avoided in the early postoperative period by having a high scleral flap resistance - scleral flap sutures are removed later when needed. Topical atropine at the end of GFS and postoperatively is useful in preventing malignant glaucoma.

In high risk eyes with cataract, a combined GFS and cataract procedure may debulk lens volume. A rigid one-piece implant may prevent a flat AC. If malignant glaucoma develops, the management is easier in a pseudophakic eye.

\section{Cataract Formation}

Cataract formation/progression may occur due to lens-corneal touch, lens trauma, inflammation, hypotony and the use of intraoperative MMC. Cataract progression is reduced by avoiding postoperative hypotony and a flat AC. The use of an oblique paracentesis minimises the risk of lens trauma. In patients with cataract, combined cataract and GFS may be considered.

\section{Ptosis and Strabismus}

Prevention

Preventative strategies include: (1) corneal traction suture rather than a superior rec-

tus suture; (2) minimise traction on the eye, which stretches the levator aponeurosis;

(3) in LBCF, dissect the conjunctiva and Tenon's as separate layers to prevent damage to the superior rectus muscle; (4) place sponges soaked in anti-fibrotic agent posteriorly as MMC is toxic to the superior rectus muscle.

Management

Conservative treatment is recommended as most cases settle spontaneously, but sometimes surgery (e.g. repair of levator dehiscence) may be required.

\section{Astigmatism}

Preventative strategies for astigmatism are: (1) minimise sclerostomy size especially

in eyes with low scleral rigidity; (2) avoid very large FBCF and scleral flaps.

\section{Conclusion}

This chapter highlights the importance of implementing a total GFS system 
start- ing from preoperative assessment, to intraoperative techniques, and finally post-operative management, which will be extensively addressed in a further chapter. It is important for surgeons to understand the principles of GFS and acquire advanced techniques to tailor GFS to each individual patient. One should be prepared to change the intraoperative strategy when unexpected conditions are encountered. Communication between glaucoma surgeon and patient is of paramount impor- tance, and management of realistic patient expectation will help ensure an optimal outcome.

\section{Acknowledgements}

Our research has been funded by the NIHR Biomedical Research Centre Moorfields Eye Hospital and UCL Institute of Ophthalmology, Fight for Sight, the Helen Hamlyn Trust, Moorfields Special Trustees, the Medical Research Council, the Guide Dogs for the Blind, the Wellcome Trust, the International Glaucoma Association, the Alcon Research Institute Prize, John Nolan, Ron and Liora Moskovitz, and the Michael and Ilse Katz Foundation.

\section{References}

1 Cairns JE: Trabeculectomy. Preliminary report of a new method. Am J Ophthalmol 1968;66:673679.

2 Gedde SJ, Schiffman JC, Feuer WJ, Herndon LW, Brandt JD, Budenz DL: Treatment outcomes in the Tube Versus Trabeculectomy Study after one year of follow-up. Am J Ophthalmol 2007;143:9-22.

3 Gedde SJ, Schiffman JC, Feuer WJ, Herndon LW, Brandt JD, Budenz DL, Tube Versus Trabeculectomy Study Group: Three-year followup of the Tube Versus Trabeculectomy Study. Am J Ophthalmol 2009;148:670-684.

4 Nilforushan N, Yadgari M, Kish SK, Nassiri N: Subconjunctival bevacizumab versus mitomycn C

adjunctive to trabeculectomy. Am J

Ophthalmol, Epub ahead of print.

5 Ang GS, Chan KC, Pootschi A, Nichoas S, Birchall W, Wakely L, Wells AP: Comparison of standard trabeculectomy versus microtrabeculectomy as a surgical treatment for glaucoma: a randomised clinical trial. Clin Experiment Ophthalmol, Epub ahead of print.

6 Stead RE, King AJ: Outcome of trabeculectomy with

mitomycin $\mathrm{C}$ in patients with advanced glaucoma.
Br J Ophthalmol 2011;95:960-965.

7 Lusthaus JA, Kubay O, Karim R, Wechsler D, Booth

F: Primary trabeculectomy with mitomycin C:

safety and efficacy at 2 years. Clin Experiment

Ophthalmol 2010;38:831-838. 
8 Reibaldi A, Uva MG, Longo A: Nine-year follow-up of trabeculectomy with or without mitomycin-c in primary open-angle glaucoma. Br J Ophthalmol 2008; 92:1666-1670.

9 Cillino S, Di Pace F, Casuccio A, Cillino G, Lodato G: Deep sclerectomy versus trabeculectomy with low-dosage mitomycin C: four-year follow-up. Ophthalmologica 2008;222:81-87.

10 Shah P, Agrawal P, Khaw PT, Shafi F, Sii F: ReGAE 7: long-term outcomes of augmented trabeculectomy with mitomycin C in African-Caribbean patients. Clin Experiment Ophthalmol, Epub ahead of print.

11 Stalmans I, Gillis A, Lafaut AS, Zeyen T: Safer trabeculectomy technique: long term outcome. Br J Ophthalmol 2006;90:44-7.

12 Khaw PT, Grehn F, Hollo G, Overton B, Wilson R, Vogel R, Smith Z: A phase III study of subconjunctival human anti-transforming growth factor beta(2) monoclonal antibody (CAT-152) to prevent scarring after first-time trabeculectomy. Ophthalmology 2007;114:1822-1830.

13 Cohen SW: Corneal traction suture. Ophthalmic Surg 1988;19:371.

14 Conklin JD, Goins KM, Smith TJ: Corneal traction suture in trabeculectomy. Ophthalmic Surg 1991;22: 494.

15 Shuster JN, Krupin T, Kolker AE, Becker B: Limbus$\mathrm{v}$ fornix-based conjunctival flap in trabeculectomy. A long-term randomized study. Arch Ophthalmol 1984;102:361-362. 
16 Wells AP, Cordeiro MF, Bunce C, Khaw PT: Cystic bleb formation and related complications in limbus- versus fornix-based conjunctival flaps in pediatric and young adult trabeculectomy with mitomycin C. Ophthalmology 2003;110:21922197.

17 Sii F, Todd B, Shah P, Chiang MY: Reduction of anterior-segment vascularity with preoperative top- ical apraclonidine 1\%. J Cataract Refract Surg 2006; 32:692-693.

18 Birchall W, Wakely L, Wells AP: The influence of

scleral flap position and dimensions on intraocular pressure control in experimental trabeculectomy. J Glaucoma 2006;15:286-290.

19 You YA, Gu YS, Fang CT, Ma XQ: Long-term effects of simultaneous subconjunctival and subscleral mitomycin $\mathrm{C}$ application in repeat trabeculectomy. J Glaucoma 2002;11:110-118.

20 Poole TR, Gillespie IH, Knee G, Whitworth J: Microscopic fragmentation of ophthalmic surgical sponge spears used for delivery of antiproliferative agents in glaucoma filtering surgery. $\mathrm{Br}$ J Ophthalmol 2002;86:1448-1449.

21 Al-Shahwan S, Edward DP: Foreign body granulomas secondary to retained sponge fragment following mitomycin C trabeculectomy. Graefes Arch Clin Exp Ophthalmol 2005;243:178-181.

22 Lieberman MF: Suture lysis by laser and goniolens.

Am J Ophthalmol 1983;95:257-258.

23 Aktan SG, Mandelkorn RM: Krypton laser suture

lysis. Ophthalmic Surg Lasers 1998;29:635-638.

24 Singh J, Bell RW, Adams A, O’Brien C: Enhancement of the post trabeculectomy bleb formation by suture lysis. Br J Ophthalmol 1996;80:624-627.

25 Haynes WL, Alward WL, McKinney JK: Lowenergy argon laser suture lysis after trabeculectomy. Am J Ophthalmol 1994;117:800-801.

26 Keller C, To K: Bleb leak with hypotony after laser suture lysis and trabeculectomy with mitomycin C. Arch Ophthalmol 1993;111:427-428.

27 Lieberman MF: Diode laser suture lysis

$$
\text { following }
$$

trabeculectomy with mitomycin. Arch Ophthalmol 1996;114:364.

28 Savage JA, Condon GP, Lytle RA, Simmons RJ: Laser suture lysis after trabeculectomy. Ophthalmology 1988;95:1631-1638.

29 Morinelli EN, Sidoti PA, Heuer DK, et al: Laser suture lysis after mitomycin C trabeculectomy. Ophthalmology 1996;103:306-314.

30 Papaa KS, Derick RJ, Weber PA, et al: Late argon laser suture lysis after mitomycin $\mathrm{C}$ trabeculectomy. Ophthalmology 1993;100:1268-1271.

31 Macken P, Buys Y, Trope GE: Glaucoma laser suture lysis. Br J Ophthalmol 1996;80:398-401.

32 Kapetansky FM: Laser suture lysis after trabeculectomy. J Glaucoma 2003;12:316-320. 
33 Schwartz AL, Weiss HS: Bleb leak with hypotony after laser suture lysis and trabeculectomy with mitomycin C. Arch Ophthalmol 1992;110:1049.

34 Raina UK, Tuli D: Trabeculectomy with releasable sutures: a prospective, randomized pilot study. Arch Ophthalmol 1998;116:12881293.

35 Shin DH, Parrow KA, Presberg-Greene SE: Tight scleral flap trabeculectomy with postoperative laser suture lysis. Am J Ophthalmol 1990;110:325.

36 Shaffer RN, Hetherington JR, Hetherington J Jr, Hoskins HD Jr: Guarded thermal sclerostomy - a preliminary report. Trans Am Ophthalmol Soc 1971;69:141-146.

37 Cohen JS, Osher RH: Releasable scleral flap suture.

Ophthalmol Clin North Am 1988;1:187-197. 38 BurchfieldJC, Kolker AE, Cook SG: Endophthalmitis

following trabeculectomy with releasable sutures.

Arch Ophthalmol 1996;114:766.

39 Rosenberg LF, Siegfried CJ: Endophthalmitis asso-

ciated with a releasable suture. Arch

Ophthalmol

1996;114:767.

40 Maberley D, Apel A, Rootman DS:

Releasable ' $\mathrm{U}$ '

suture for trabeculectomy surgery. Ophthalmic Surg

1994;25:251-255.

41 Kolker AE, Kass MA, Rait JL: Trabeculectomy

with releasable sutures. Arch Ophthalmol 1994;112:

62-66.

42 Cohen JS, Osher RH: Endophthalmitis associated

with releasable sutures. Arch Ophthalmol 1997;115:

292.

43 Foster PJ, Wilkins M, Khaw PT: Trabeculectomy

with releasable sutures - a modified technique. Asia

P J Ophthalmol 1996;8:13-16.

44 Ashraff NN, Wells AP: Transconjunctival suture

adjustment for initial intraocular pressure control

after trabeculectomy. J Glaucoma 2005;14:435440.

45 Wells AP, Bunce C, Khaw PT: Flap and suture

manipulation after trabeculectomy with adjustable

sutures: titration of flow and intraocular pressure in guarded filtration surgery. J Glaucoma 2004;13: 400-406.

46 AGFID: Experimental flow studies in glaucoma

drainage device development. Br J Ophthalmol 2001; 85:1231-1236.

47 Wise JB: Mitomycin-compatible suture technique

for fornix-based conjunctival flaps in glaucoma filtration surgery. Arch Ophthalmol 1993;111: 992-997.

48 Auw-Haedrich C, Funk J, Boemer TG: Long-term results after filtering surgery with limbal-based and fornix-based conjunctival flaps. Ophthalmic Surg Lasers 1998;29:575-580.

49 Hitchings and Grierson: Clinico-pathological cor-

relation in eyes with failed fistulizing surgery. Trans Ophthalmol Soc U K 1983;103:84-88. 
50 DeBry PW, Perkins TW, Heatley G, Kaufman P, Brumback LC: Incidence of late-onset blebrelated complications following trabeculectomy with mito- mycin. Arch Ophthalmol 2002;120:297-300.

51 Zacharia PT, Deppermann SR, Schuman JS: Ocular hypotony after trabeculectomy with mitomycin C. Am J Ophthalmol 1993;116:314-326.

52 Bindlish R, Condon GP, Schlosser JD, D’Antonio J, Lauer KB, Lehrer R: Efficacy and safety of mitomycin-C in primary trabeculectomy: five-year follow- up. Ophthalmology 2002;109:1336-1341.

53 Fechtner RD, Minckler D, Weinreb RN, et al: Complications of glaucoma surgery. Ocular decom- pression retinopathy. Arch Ophthalmol 1992;110:

965-968.
54 Suzuki R, Nakayama M, Satoh N: Three types of retinal bleeding as a complication of hypotony after trabeculectomy. Ophthalmologica 1999;213:

135-138.

55 Grunwald JE, Riva CE, Stone RA, et al: Retinal autoregulation in open-angle glaucoma.

Ophthalmology 1984;91:1690-1694.

56 Minckler DS, Bunt AH: Axoplasmic transport in ocular hypotony and papilledema in the monkey.

Arch Ophthalmol 1977;95:1430-1436.
Prof. P.T. Khaw

National Institute for Health Research

Biomedical Research Centre

Moorfields Eye Hospital and UCL Institute of Ophthalmology

Eyes and Vision UCL Partners and UCL School of Pharmacy

11-43 Bath Street

London EC1V $9 \mathrm{EL}$ (UK)

Tel. +44 207608 6887, E-Mail p.khaw@ucl.ac.uk 